\title{
A model experiment to study swallowing of spherical and elongated particles
}

\author{
Marco Marconati ${ }^{1, \star}$, Sharvari Raut ${ }^{1}$, Farshad Charkhi $^{1}$, Adam Burbidge $^{2}$, Jan Engmann ${ }^{2}$, and Marco Ramaioli ${ }^{1}$ \\ ${ }^{1}$ Department of Chemical and Process Engineering, University of Surrey, Guildford GU2 7XH, UK. \\ ${ }^{2}$ Nestlé Research Center, Route du Jorat 57, 1000 Lausanne 26, Switzerland.
}

\begin{abstract}
Swallowing disorders are not uncommon among elderly and people affected by neurological diseases. For these patients the ingestion of solid grains, such as pharmaceutical oral solid formulations, could result in choking. This generally results in a low compliance in taking solid medications. The effect of the solid medication size on the real or perceived ease of swallowing is still to be understood from the mechanistic viewpoint. The interplay of the inclusion shape and the rheology of the liquid being swallowed together with the medication is also not fully understood. In this study, a model experiment was developed to study the oropharyngeal phase of swallowing, replicating the dynamics of the bolus flow induced by the tongue (by means of a roller driven by an applied force). Experiments were performed using a wide set of solid inclusions, dispersed in a thick Newtonian liquid. Predictions for a simple theory are compared with experiments. Results show that an increase in the grain size results in a slower dynamics of the swallowing. Furthermore, the experiments demonstrated the paramount role of shape, as flatter and more streamlined inclusions flow faster than spherical. This approach can support the design of new oral solid formulations that can be ingested more easily and effectively also by people with mild swallowing disorders.
\end{abstract}

\section{Introduction}

Ingestion of grains and solid oral medications can be particularly challenging for people affected by swallowing disorders. While aspiration of liquids can expose the patients to the risk of aspiration pneumonia, swallowed solids could potentially become trapped in the larynx folds, hence leading to a potential risk of choking. Moreover, retained solid oral formulations may even start dissolving in the pharyngeal and upper esophageal regions and cause local injuries resulting in pain, esophagitis and ultimately ulceration, stricture, and perforation if the dissolved medicine irritates the mucosa of the esophagus [1]. Size and shape are deemed the most important reason that limit acceptability of prescriptions among the individuals who find issues in taking tablets and capsules [2]. In particular the insurgence of compliances, such as aspiration and choking during the oropharyngeal phase of deglutition, becomes increasingly common as the size of the oral prescription is increased [3]. Indeed studies reveal that the level of acceptability of an oral medication, albeit dependent upon the age subset, is greatly reduced when its diameter is above $8 \mathrm{~mm}$ [4]. It has also been shown that size is a major player in determining the esophageal transit time, smaller tablets flowing faster. however, there is a lack of quantitative studies on the duration of the oropharyngeal phase of swallowing and the effect of the size of the oral prescription. Other than size, shape is also an important factor to be taken into account in pill design. Previous

\footnotetext{
^e-mail: m.marconati@surrey.ac.uk
}

studies suggest that oval tablets may be easier to swallow and have faster esophageal transit times than round tablets of the same weight [5]. However, it was shown that flat tablets have greater tendency to adhere and stick to the esophageal epithelium than capsule-shaped tablets [6], hence potentially leading to esophagitis. Finally, factors such as density and pill surface characteristics may all affect the swallowability and esophageal transit of tablets and capsules. This study considered the oral transit time of tablets and pills to quantify the importance of size and shape. In order to lay the basis for a mechanistic understanding of these phenomena, before moving to panel tests, the study has been carried out using an in vitro model of the oral cavity. The same model was previously successfully used to evaluate the effect of bolus rheology on its flow and those results were successfully compared with in vivo ultrasound observations [7].

\section{Materials and Methods}

Different sizes and shapes of solid inclusions were used in the experiments. The effect of size is investigated using calibrated plastic beads, of diameters ranging from 4.8 to $10 \mathrm{~mm}$. Commercially available capsules and a pharmaceutical tablet were also later employed to study the effect of shape on the kinematics of the bolus. Empty capsules size 00 and size 3 were purchased and filled with sucrose so that their density would match that of the polyethylene beads $\left(0.875 \pm 0.050 \mathrm{~kg} \mathrm{~m}^{-3}\right)$. The size 00 capsule was used as a limiting case, as it is currently the maximum size 
Table 1. List of the solid inclusions dispersed in glycerol and used during the experiments with the in vitro model

\begin{tabular}{lll}
\hline Type of inclusion & Size & Weight \\
\hline sphere & $\oslash=4.8 \mathrm{~mm}$ & $0.049 \mathrm{~g}$ \\
sphere & $\oslash=6.35 \mathrm{~mm}$ & $0.116 \mathrm{~g}$ \\
sphere & $\oslash=8 \mathrm{~mm}$ & $0.225 \mathrm{~g}$ \\
sphere & $\oslash=10 \mathrm{~mm}$ & $0.462 \mathrm{~g}$ \\
capsule size 00 & $L=23.5 \mathrm{~mm} \oslash=8.5 \mathrm{~mm}$ & $0.912 \mathrm{~g}$ \\
capsule size 3 & $L=15.5 \mathrm{~mm} \oslash=5.8 \mathrm{~mm}$ & $0.246 \mathrm{~g}$ \\
ellipsoidal tablet & $\mathrm{H}=5 \mathrm{~mm} \mathrm{~L}=\mathrm{W}=11 \mathrm{~mm}$ & $0.436 \mathrm{~g}$ \\
\hline
\end{tabular}

for a generic drug suggested by the Food and Drug Administration. The size 3 capsule was instead specifically chosen because it has the same volume and mass of a $8 \mathrm{~mm}$ bead, hence allows for direct comparison of shape effect. Finally, an ellipsoidal uncoated tablet was used to provide a preliminary assessment of the relative deviation of the other objects from a reference over the counter pain relief. The inclusion dimensions and volume are reported in Tab. 1.In all cases the solids were dispersed in $99.98 \% \mathrm{w} / \mathrm{w}$ glycerol (SG 1.25, $\mu=1.05$ ) that was used throughout the experimental campaign as suspending liquid carrier.

\subsection{The in vitro model experiment}

In this study an in vitro model experiment is used to replicate the essential features of the peristaltic flow, observed in vivo with both ultrasound and video fluoroscopy measurements. The oral cavity is substituted with a thin plastic membrane that sticks to the upper palate. The liquid bolus is driven to flow by a rigid roller deforming the lower boundary of the membrane. The roller movement mimics the wave of muscular contraction traveling from the front to the back of the tongue. A set of calibrated weights is used to modulate the applied driving force (see Fig. 1). The occlusion is complete. Moreover, the associated wavenumber, defined by the ratio of bolus radius to its length is on the order of 0.15 . During the study, three different applied forces were investigated, by attaching the masses $m=200,270$, and $400 \mathrm{~g}$ to the driving pulley (approximately 2, 2.7, and $4 \mathrm{~N}$ ). Using these three forces resulted in maximum linear velocities of the bolus below 1 $\mathrm{m} / \mathrm{s}$ thereby the Reynolds number, defined as $R e=R v / v$, resulted to be less than 0.5 . Whilst previous experimental and numerical studies already considered similar Reynolds numbers and wavenumber [11] [10] and the propagation of a single peristaltic wave over a linear trajectory, the peristaltic transport of macroscopic solid inclusions at amplitude ratios close to unity had not been studied, as far as the authors are aware. The total inertia of the rotating arm was chosen to match the in vivo the ultrasound measurements obtained in a previous study [7]. In the experimental setup the roller movement is triggered under the release of a pin and the experiment is then recorded using a fast camera (Model ac1920-155 $\mu \mathrm{m}$, Blaser, Germany) at acquisition rate of 150 frame per second (fps). Image processing tools are subsequently employed to extract from each frame the position of the roller with respect of the center of rota-

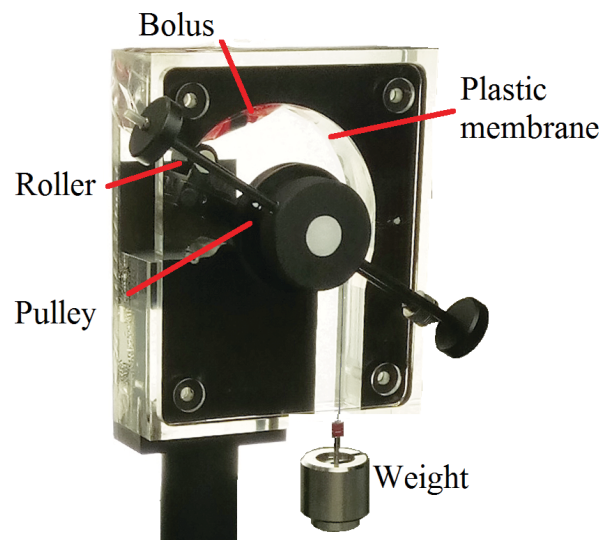

Figure 1. Schematics of the in vitro setup.

tion, and the solid inclusion position. Velocity profiles are obtained by means of raw data filtering, followed by threepoint numerical differentiation of the instantaneous roller angular positions. The velocity profiles were compared with the theoretical values obtained solving the differential equation describing the motion in absence of solid inclusions. The relative displacement between the solid inclusion and the bolus was quantified by the difference of the instantaneous roller and bead angular positions.

\subsection{Theory}

Previous studies considered in vitro swallowing of homogeneous liquids, either Newtonian or shear thinning, to address swallowing disorders [8] [7]. The acceleration and motion of the bolus was also successfully described by a simple mathematical model, considering the torque generated by the applied force, the inertia of the system and the drag force generated by the liquid bolus flow. The viscous dissipation was computed based on a modified Hagen-Poiseuille flow, corrected by a factor $\beta$ whose value is independent on the applied force to the system and the viscosity of the liquid. This theory has then been modified to account for suspended solids, considering the apparent restriction in the effective longitudinal section of the bolus experienced in proximity of the hard inclusion. The flow field was thereby simplified, considering an annular flow of length $L=L_{\text {bolus }}$, where the inner shell has a diameter equivalent to the inclusion considered $\left(R_{i}\right)$, while the outer shell diameter is fixed and correspondent to the diameter of the bolus $\left(R_{e}\right)$. Boundary conditions on velocity apply at the walls: the inner cylinder (i.e. the inclusion) was supposed to move longitudinally, in the direction of swallowing, with velocity equal to to that of the roller $v_{\text {roller }}$, while a no-slip condition applies to the external shell (i.e. the plastic membrane in which the bolus is contained). A similar problem was previously considered by Charles in the modeling of pipeline flow of cylindrical capsules [12]. The theory there presented, validated on experiments at low Reynolds numbers [13], indicates that the pressure drop experienced in transporting a infinitely long cylindrical neutrally buoyant object without axial offset, can be 


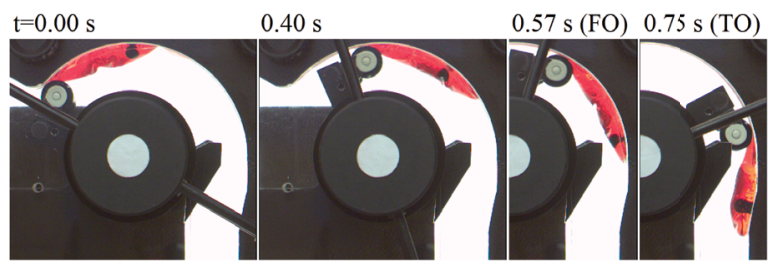

Figure 2. Screenshots of the in vitro swallowing of a single plastic sphere of $6.35 \mathrm{~mm}$ diameter suspended in glycerol at external applied force of $2 \mathrm{~N}$.

related to the pressure drop in a Poiseuille flow, with identical mean velocity, corrected by a factor dependent upon of the ratio of radii $k=R_{i} / R_{e}$ of the inclusion and the pipe. On this basis, the same correction was applied to relate the viscous dissipation of the bolus flow in presence of solid inclusions to that of the homogeneous fluid in a Hagen-Poiseuille flow.

$$
F_{d}^{*}=\left(1-k^{4}\right)^{-1} \beta 8 \pi \mu L v_{\text {roller }}
$$

Eq. 1 correctly reduces to the theory presented by Hayoun et al. [7] when the cross sectional radius of the inclusion in the direction perpendicular to the flow is reduced $\left(R_{i} \rightarrow 0\right)$.

\section{Results}

The objective of the experiments was to understand the effect of inclusion size and shape on the flow, when varying the applied force. Some characteristic events during the bolus flow are illustrated in Fig. 2, in the case of a $6.35 \mathrm{~mm}$ spherical inclusion swallowed at the lowest applied force $(2 \mathrm{~N})$. The event FO and TO are of particular importance, as they represent respectively the exit of the front of the bolus from the plastic tube (Front Out - FO), and the moment in which the roller reaches the final position that corresponds to the tail of the bolus leaving the tube (Tail Out TO). The time when these events occur depends on the size and shape of inclusions and on the applied load. The evolution of the roller angular velocity in time, till bolus front out obtained for different inclusions and applied loads is illustrated in Fig. 3. For a given applied force, lines of different colors represent the experimental results different diameters of spherical inclusions. These can be also compared with the theoretical curves (thin solid lines), obtained solving the rotational trajectory of the system, using the viscous dissipation described in Eq. 1. The theory predicts a monotonic increase of roller angular velocity with time. This had been compared favorably against in vitro experiments carried out in absence of any solid inclusion [7]. In these conditions, an initial inertial regime was observed where the angular velocity is increasing linearly with time, followed by a viscosity-dominated regime, in which the drag force becomes predominant and the bolus velocity becomes constant. Conversely, experiments either at higher applied loads, or lower viscosities, only showed the inertial regime. The velocity profiles of the experiments with inclusions, as shown in Fig. 3, generally exhibit similar features. In particular, the inertial regime, below $t=0.1 \mathrm{~s}$, is still well predicted by the theory and the theoretical curves are close to the initial slope of the experimental data. Experiments at reduced external forces ( $2 \mathrm{~N}$ ) have been previously demonstrated to best match in vivo ultrasound observations [8] and are therefore the conditions closest to a human swallow. The in vitro results show that angular velocity decreases, when increasing the size of the spherical inclusion. This is well reproduced by the theory, in particular in the case of the biggest inclusion (spherical bead $d=10 \mathrm{~mm}$ ), when the velocity approaches a constant asymptote in the viscous regime. The experimental velocity profiles obtained using the highest load configuration $(4 \mathrm{~N})$ show no significant effect of the inclusion size. The theory captures well the experimental curves, with the exception for the biggest inclusion, where the theory over predicts the viscous dissipation. The intermediate load $(2.7 \mathrm{~N})$ shows a trend similar to the lower load and the $10 \mathrm{~mm}$ inclusion dynamics is significantly slower than the others. Inclusion shape has also a paramount impact on bolus kinematics, as can be appreciated comparing the profiles for spherical and elongated objects (Fig. 4). The capsule size 3 has almost identical volumes and mass (Table 1) than that of the $d=8$ $\mathrm{mm}$ sphere, yet the capsule flows significantly slower than the bead. Conversely, the ellipsoidal tablet is heavier and longer than the $d=10 \mathrm{~mm}$ bead, but it flows as fast as a $d=8 \mathrm{~mm}$ bead. In all cases the theoretical values obtained using the inclusion cross section as $R_{i}$ in Eq. 1 over predicts the velocity profile. This suggests that more complex models are required to account realistically for the inclusion shape. The relative motion the solid inclusion within the bolus was measured using image analysis. This can clarify the deviation from considering that the velocity of the inclusion $v_{i}$ equals the linear velocity of the roller, as assumed in the computation of the viscous dissipation term of Eq. 1. The profiles of $\Delta \alpha$ as a function of time highlight a noticeable reduction in the angular distance between the inclusion and the roller in both the extremes cases of 4.8 $\mathrm{mm}$ and $10 \mathrm{~mm}$ bead diameters. In those cases angular deviations of up to $-30^{\circ}$ from the initial position are experienced. Conversely, no significant trends are observed in the case of the intermediate size beads $(6.35$ and $8 \mathrm{~mm})$, the size 3 capsule, and the ellipsoidal tablet. The backward motion of the smallest solid inclusion is of particular interest, as it confirms the fact that small objects are less efficiently transported by peristalsis [10]. On the other hand, the fact that the $10 \mathrm{~mm}$ bead is also moving backwards in the bolus frame of reference, is mainly deemed to the non negligible wall friction experienced when running experiments with the biggest inclusions. Increasing the external applied load up to $4 \mathrm{~N}$ does not alter the bead relative position: the $4.8 \mathrm{~mm}$ and the $10 \mathrm{~mm}$ beads consistently move backwards, while no noticeable variations in bead position are observed for both the 6.35 and the $8 \mathrm{~mm}$ beads. The measured velocity of the inclusion is therefore always lower or equal to that of the roller. Furthermore, the less effective peristaltic transportation of smaller in- 


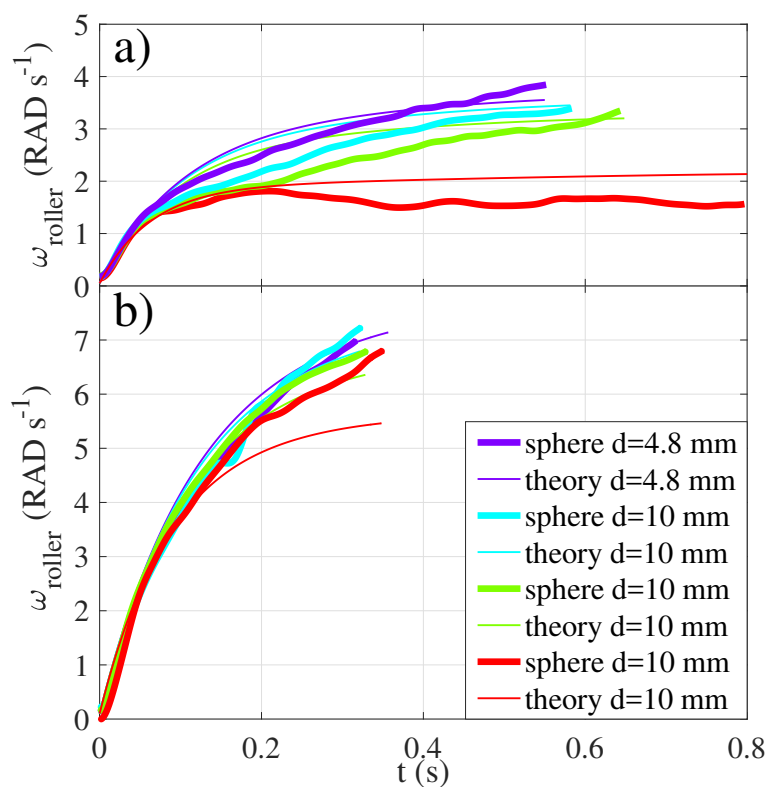

Figure 3. Evolution with time of the angular velocity of the tail of the bolus for different diameters of spherical inclusions and two applied forces: a) $2 \mathrm{~N}$, b) $4 \mathrm{~N}$. Theoretical values are illustrated with thin solid lines.

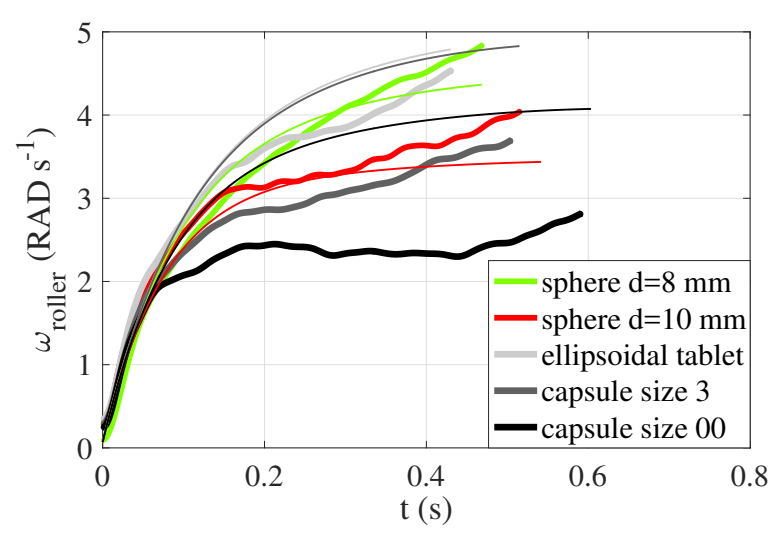

Figure 4. Evolution of the roller velocity with spherical and nonspherical inclusions at $2.7 \mathrm{~N}$ load. Thin lines represent the theoretical values obtained assuming $R_{i}$ equal to the cross sectional radius of the inclusion in Eq. 1.

clusions is of particular interest from the clinical prospective, as it highlights the need for further liquid swallows to transport the hard object into the esophagus.

\section{Conclusions}

Building on the results obtained in previous studies [7] and [8], this article proposes a novel approach to study the swallowing dynamics of hard particles in liquids with high viscosity during the oropharyngeal phase of human swallowing. Spherical and non-spherical inclusions in size ranging 4.8 to $10 \mathrm{~mm}$ were considered. The experimental results demonstrated that inclusions affect significantly the peristaltic flow, in particular at low applied forces. Inclusion size influences bolus velocity: the bigger the diameter of the inclusion, the longer the oral transit time. A simple mathematical model was used to successfully evaluate the viscous dissipation at very low Reynolds numbers for big enough inclusion, although the drag force is over predicted at higher applied loads. The shape of the inclusion influences the dynamics of the system too. Inclusions of same volumes and mass, but significantly different shape show a different oral transit times. Finally, the efficiency of peristaltic transport is higher for inclusion of intermediate size while both small beads and very large inclusions flow backwards, toward the trailing edge of the bolus. This phenomenon could potentially lead to residues of inclusions in the pharynx. These results on the oral phase of swallowing of hard solid inclusions, can support the development of novel pill designs, before costly and invasive clinical trials.

\section{References}

[1] F. J. Collins, H. R. Matthews, S. E. Baker, J. M. Strakova, Brit. Med. J. 2, 132-133 (1979).

[2] FDA guidance for industry, Size, shape, and other physical attributes of generic tablets and capsules (US FDA, Rockville, MD, 2015).

[3] J. Kelly, G. D’Cruz, D. Wright, J. Adv. Nurs. 66, 8291 (2010).

[4] D. P. Brotherman, T. O. Bayraktaroglu, R. J. Garofalo, J. Am. Pharm. Ass. 44, 587-593 (2004).

[5] H. Hey, F. Jorgensen, K. Sorensen, H. Hasselbelch, T. Wamberg, Brit. Med. J. 285, 1717-1719 (1982).

[6] M. Marvola, M. Rajaniem, E. Marttila, K. Vahervuo, A. Sothmann, J. Pharm. Sci. 72, 1034-1036 (1983).

[7] P. Hayoun, J. Engmann, S. Mowlavi, B. Le Reverend, A. Burbidge, M. Ramaioli, J. Biomech. 48, 3922-3928 (2015).

[8] S. Mowlavi, J. Engmann, A. Burbidge, R. Lloyd, P. Hayoun, B. Le Reverend, M. Ramaioli, J. Biomech. DOI 10.1016/j.jbiomech.2016.10.011 (2016).

[9] H. Chisaka, Y. Matsushima, F. Wada, S. Saeki, Dysphagia 21, 275-279 (2006).

[10] T. K. Hung and T. D. Brown, J. Fluid Mech. 73, 77-96 (1976).

[11] L. J. Fauci, Comput. Fluids 21, 583-598 (1992).

[12] M. E. Charles, Can. J. Chem. Eng. 41, 46-51 (1963).

[13] H. S. Ellis, Can. J. Chem. Eng. 42, 1-8 (1964). 